\title{
Al-Driven Sales Automation: Using Chatbots to Boost Sales
}

\author{
Christian Hildebrand and Anouk Bergner
}

Chatbots - the new sales agents? $\times$ Advances in artificial intelligence and natural language processing are giving rise to a new kind of service encounter: chatbots. These humanized interfaces allow brands to provide $24 / 7$ support while automating major areas of customer service operations. Recent industry reports indicate that more than $80 \%$ of all businesses are considering integrating chatbots within the next five years. Many service providers are already using chatbots, but most applications have focused on cost saving and automation to partially replace human service encounters. However, rather than a mere cost-saving opportunity, chatbots might bring a myriad of advantages in automating customer-company interactions. One critical question for business is: Can chatbots augment the corporate sales force? More directly, are chatbots able to sell?

Through a series of field and laboratory studies, we found evidence that chatbots can indeed alter consumer preferences and purchase decisions. What consumers enjoy in particular is the ability to engage in natural dialogue and even to connect to the chatbot's "personality." We gained insights on the "optimal mechanics" of such interfaces and found evidence that incorporating chatbots into consumers' shopping processes promotes more intimate consumer-brand relationships, greater trust, and can be used as a powerful opportunity to upsell.
KEYWORDS

Chatbots, Sales Automation, Turn-Taking, Personalization, Trust

THE AUTHORS

\section{Christian Hildebrand}

Director and Professor of

Marketing Analytics,

Institute of Marketing (IfM-HSG),

University of St. Gallen,

Switzerland

christian.hildebrand@unisg.ch

\section{Anouk Bergner}

University of St. Gallen, Switzerland

Anouk.Bergner@unisg.ch 





BOX 1

\section{Key ingredients to design better chatbot touchpoints}

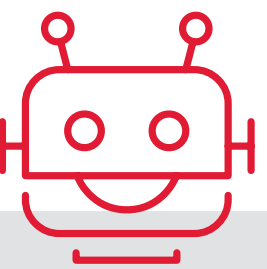

To assess the dynamics of the psychological mechanisms of turn-taking and personalization, we built different types of chatbot interfaces that varied in their ability to emulate natural dialogue and their level of personalization. Our participants interacted with these interfaces in different industries, like car rental services, financial advisory and online fashion retail.

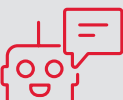

> We found that the turn-taking mechanism and the ability to react naturally to a user's input are the foundational building blocks for more intimate and trusting brand relationships and more positive brand attributions.



Our studies revealed a significant impact of the formality of the language employed by the interface. More informal communication styles, using emojis and trivial acknowledgements like "Great, thanks!" following a consumer input, significantly enhanced close consumer-brand relationships.

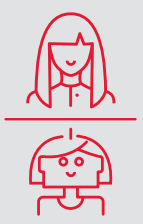

$>$ We also found that chatbots mirroring even generic consumer characteristics significantly increased its persuasion potential. Merely matching a consumer's gender or using a similar sounding name led to increased upselling compared to traditional interfaces (i.e., websites).

Sales automation with chatbots: The critical role of turntaking and personalization $`$ First, a chatbot's ability to engage in more natural dialogue compared to traditional media is a critical ingredient for fostering a more enjoyable, trusted user experience. Research in human-to-human interaction has shown that turn-taking during conversations makes the conversational partners feel closer and that they like each other more. Thus, turn-taking is key to fostering trust between interaction partners.

The second important driver for building closer consumerbrand relationships is personalization. Both in terms of the chatbot being viewed as more human-like compared to a traditional interface as well as in its ability to adapt to specific consumer characteristics. People see and treat machines like other humans when these machines possess more human-like features. Brands and products with more human characteristics also lead to more positive brand association and more intimate brand relationships, according to prior research.
However, apart from the obvious visual characteristics of a chatbot, it is not very clear what makes an interaction with a machine "feel human" and personal, and how this ultimately affects consumer purchase decisions. A condensed summary of our key insights is presented in Box 1.

The powerful impact of chatbots upsell potential $`$ In the context of our car rental bot, we found that consumers were almost twice as willing to trade-up to more costly options and add-on services when they were offered by a humanized chatbot (see Figure 1). This effect increased even further for more personalized interfaces matching fundamental consumer characteristics, according to additional experiments. 


\section{$\gg$ \\ Incorporating chatbots into consumers' shopping \\ processes promotes more intimate consumer-brand \\ relationships, greater trust, and can be used as \\ a powerful opportunity to upsell.}

$\ll$

FIGURE 1 > Willingness to trade up to a more costly car rental option

depending on bot-type

\section{Choice Upsell Option}



The powerful impact of chatbots on trust $\rtimes$ In our work with a financial advisory chatbot, known as a "robo advisor," we found astonishing results with people putting too much trust in recommendations provided by robo advisors. We went as far as proposing objectively wrong portfolio recommendations to knowledgeable investors. Despite the objectively wrong advice and additional warnings to investors, they were three times more likely to accept an incorrect recommendation from a humanized robo advisor than from a traditional web interface. Figure 2 clearly depicts the greater trust in the robo-advisors and substantially fewer rejections of the recommended portfolio. Red dots indicate rejection of the recommended portfolio.

In another study, allowing consumers to customize a dress shirt via a humanized chatbot rather than a traditional e-commerce website also led to a significant increase in customers' willingness to pay for the custom-made dress shirt.
Designing better chatbot touchpoints $\varkappa$ The implementation of bot interfaces varies tremendously in current industry practice. They range from human-like bot interfaces to merely presenting a brand logo or a digital avatar. Some applications provide a maximum amount of information with limited turn-taking between the user and the interface, others offer only short pieces of information and require more turn-taking. Often, the implementation is a function of the default settings provided by chatbot providers and platforms. Instead of simply implementing the default option, companies should consider very carefully how the specifics of the chatbot interface might affect the user experience.

Our work provides clear guidelines for developing better customer-bot touchpoints - for both customers and companies. Four effective design "mechanics" may serve as a guideline. 
FIGURE $2>$ Trust perceptions and rejection of an objectively incorrect

\section{portfolio recommendation}

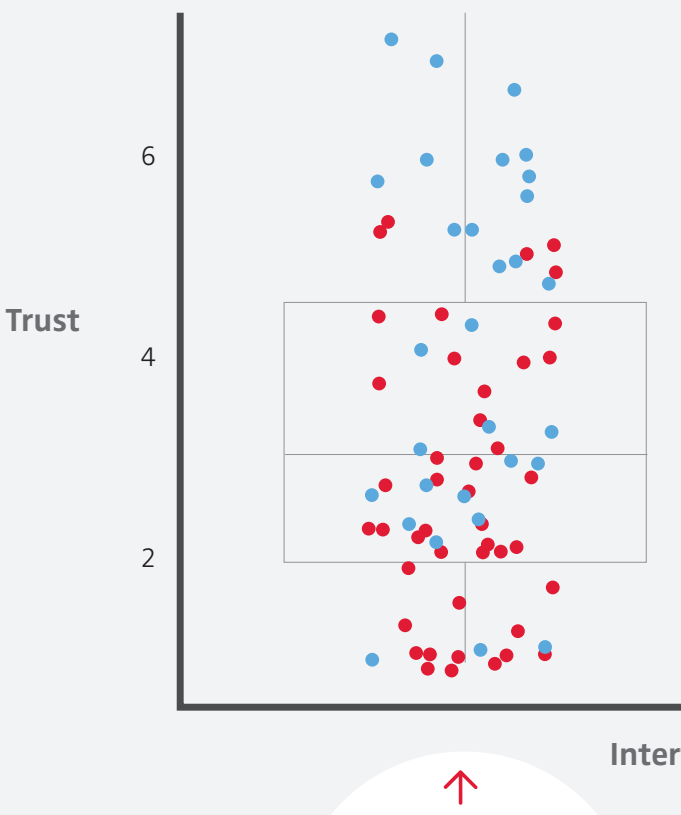

Website

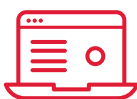

Recommendation accepted

Recommendation rejected
$>$ Make the interaction feel natural $\ltimes$ Our studies reveal that greater turn-taking between the user and the interface is an important lever for generating trust. This critical trust-building mechanism has positive downstream consequences on brand perception and upselling potential. Managers should move beyond "out-of-the-box" chatbot services. Simple mechanics such as increasing the frequency of interactions leads to greater trust and a more enjoyable user experience. Yet, managers should carefully monitor and detect the flow of the conversation and provide witty answers to random and unrelated questions. Should the conversation with the chatbot get stuck nevertheless, companies should have hybrid systems at the ready that quickly connect users to a human representative.
$>$ Adapt chatbots to consumer characteristics $`$ While we have only investigated a few ways of personalizing chatbots - based on consumers' gender, using similarsounding first names, mimicking the type of language used - we see great potential for brands in providing a personalized chatbot experience. Our findings suggest that personalizing chatbots to basic consumer characteristics increases trust perception and improves the perception of intimacy between the customer, the chatbot and, ultimately, the brand.

$>$ Adapt chatbots to your brand $`$ A chatbot should not only adapt to consumer characteristics, but it should also reflect the key characteristics of the brand. While we did 


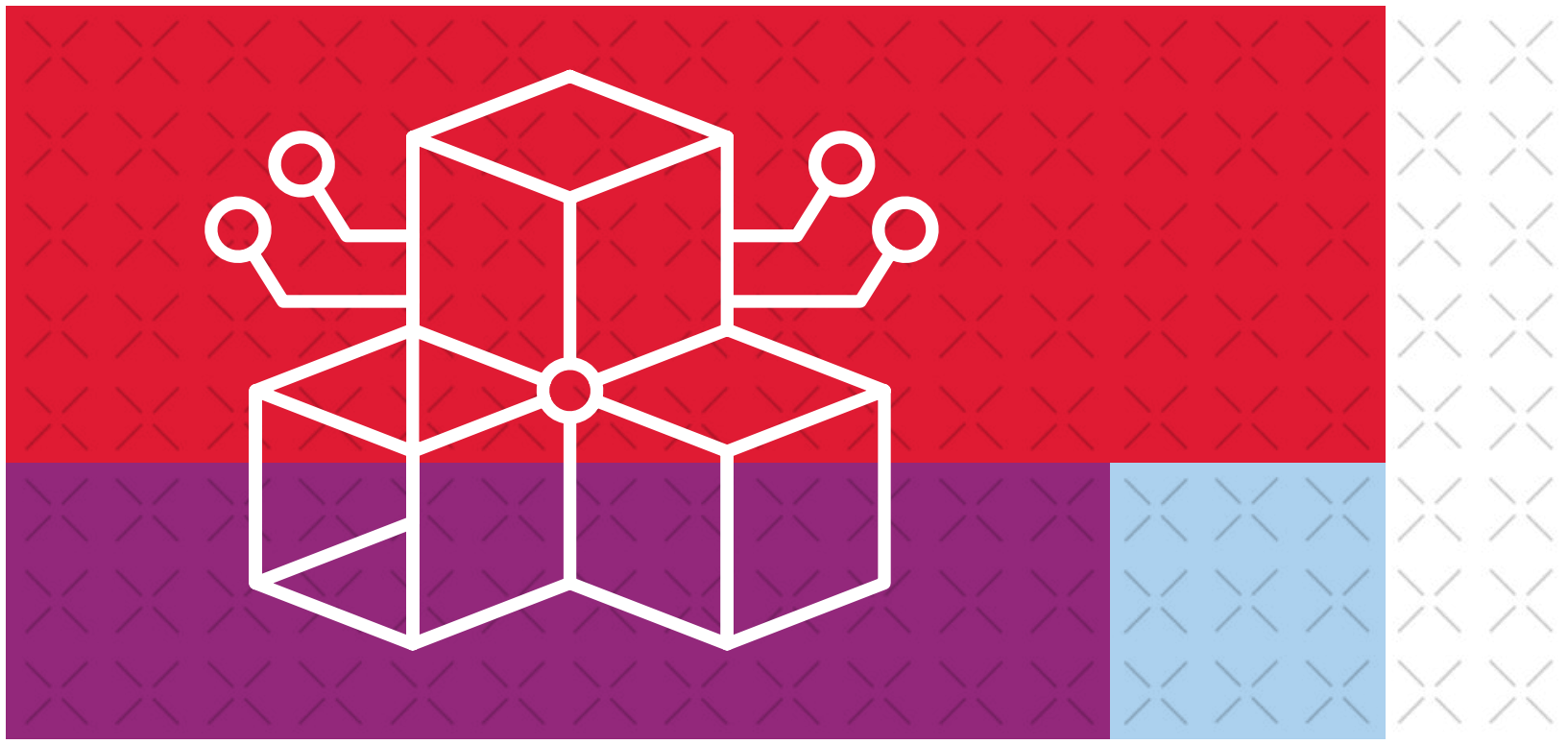

not investigate this aspect in this research, we expect that chatbot "personalities" can be specifically tailored to the desired brand personality. Not only structural aspects of a chatbot interaction like the extent of turn-taking, but also more contextual factors such as the personality expressed through the type of language will affect both brand perception and consumer choice. Thus, designing the personality of machines more generally will be an exciting avenue for future research and brand strategists alike.

$>$ Beware of the potential downsides $`$ Finally, consider the potential downsides and pain points for users. As illustrated in the article by Hadi and colleagues in this issue, there can be severe downsides to the humanization of chatbots in certain applications. They show that providing automated service interactions to already dissatisfied customers can create a downward spiral that hurts both the entire service value chain and the brand.

\section{Chatbots as persuasion agents: Augmenting the cor-} porate salesforce $\ltimes$ Will machine intelligence improve enough that consumers will no longer be able to distinguish a machine from a human? As developments in artificial intelligence advance at an ever-increasing rate, it is not inconceivable that chatbots and other humanized interfaces will reach this benchmark in the near future. Brand managers should therefore consider chatbots not merely as another digital marketing fad or a way to save costs through service automation. Chatbots can be a powerful tool in fundamentally changing the orchestration of a brand's digital touchpoints and ultimately step-change a company's sales potential. Possible applications go beyond mere service interactions and can have direct effects on a company's top-line growth.
Chatbot personas have the potential to persuade customers, to offer extended features, to sell upgrades and build closer customer-brand relationships. Companies seeking to leverage the full potential of $\mathrm{Al}$ in the context of conversational interfaces and digital assistants should not overlook the critical process of optimizing the design aspects of their chatbots rather than simply using "off-the-shelf" chatbot templates. 冫
FURTHER READING

Bergner, A.; Hildebrand, C. and Häubl, G. (2018): "Machine Talk: How Conversational Interfaces Promote Brand Intimacy and Influence Consumer Choice", Association for Consumer Research, Conference Proceedings.

Dale, R. (2016): "The Return of the Chatbots", Natural Language Engineering, 22(5), 811-17.

Hildebrand, C. and Bergner, A. (2019): “Detrimental Trust in Automation: How Conversational Robo Advisors Leverage Trust and Miscalibrated Risk Taking", Society for Consumer Psychology, Conference Proceedings.

Levinson, S. C. (2016): "Turn-taking in Human Communication - Origins and Implications for Language Processing", Trends in Cognitive Sciences, 20 (1), 6-14. 